\title{
Efeito dos Surfactantes DSS e LAS-C12 sobre o Camarão-rosa (Farfantepenaeus paulensi, Pérez-Farfante, 1967)
}

\author{
E. BARBIERI \\ Instituto de Pesca da Secretaria da Agricultura e Abastecimento do \\ Estado de São Paulo, C.P. 61, CEP 11990-000, Cananéia, SP
}

(Received March 2, 2007; Accepted December 12, 2007)

\begin{abstract}
RESUMO
Neste trabalho avaliou-se a toxicidade aguda do DSS (Dodecil Sulfonato de Sódio) e do LAS-C12 (Dodecil Benzeno Sulfonato de Sódio Linear) utilizando camarões-rosa (Farfantepenaeus paulensis) expostos a diferentes concentrações, por um período de até 96 horas. A toxicidade aguda do DSS para larvas do camarão-rosa ( $F$. paulensis) expostas a diferentes concentrações foi: $13,66 \mathrm{mg} \mathrm{L}^{-1}$, para o período de exposição de 24 horas; $11,89 \mathrm{mg} \mathrm{L}^{-1}$, para 48 horas; $9,98 \mathrm{mg} \mathrm{L}^{-1}$, para 72 horas; e 8,66 $\mathrm{mg} \mathrm{L}^{-1}$, para 96 horas, respectivamente. Já para o LAS-C12 situaram-se em: 6,37 $\mathrm{mg} \mathrm{L}^{-1}$, para o período de exposição de 24 horas; $5,32 \mathrm{mg} \mathrm{L}^{-1}$, para 48 horas; 4,61 $\mathrm{mg} \mathrm{L}^{-1}$, para 72 horas; e 4,25 $\mathrm{mg} \mathrm{L}^{-1}$, para 96 horas, respectivamente.
\end{abstract}

Palavras-chave: camarão-rosa, LAS-C12, DSS toxicidade, Farfantepenaeus paulensis, detergentes.

\section{ABSTRACT \\ Effects of surfactants DSS and LAS-C12 on pink shrimp (Farfantepenaeus paulenssi, Pérez-Farfante, 1967)}

An evaluation was done of acute toxicity of the SDS (Sodium Dodecil Sulfonate) and LAS-C12 (Linear Alkylbenzene Sulfonate) using red shrimp (Farfantepenaeus paulensis) exposed to different concentration, for periods of up to 96 hours. The acute toxicity of SDS in larvae of the pink shrimp exposed to different concentrations of this substance were: 13,66 mg $\mathrm{L}^{-1}$ for $24 \mathrm{~h}$ of exposure; $11.89 \mathrm{mg} \mathrm{L}^{-1}$ for $48 \mathrm{~h} ; 9.98 \mathrm{mg} \mathrm{L}^{-1}$ for $72 \mathrm{~h}$, and $8.66 \mathrm{mg} \mathrm{L}^{-1}$ for $96 \mathrm{~h}$. For LAS-C12 the acute toxicity in larvae of the pink shrimp exposed to different concentrations of this substance, for period of up to $96 \mathrm{~h}$ were 6.37 $\mathrm{mg} \mathrm{L}^{-1}$ for $24 \mathrm{~h}$ of exposure; $5.32 \mathrm{mg} \mathrm{L}^{-1}$ for $48 \mathrm{~h}$; $4.61 \mathrm{mg} \mathrm{L}^{-1}$ for $72 \mathrm{~h}$; and $4.25 \mathrm{mg} \mathrm{L}^{-1}$ for $96 \mathrm{~h}$.

Key words: pink shrimp, LAS-C12, SDS, toxicity, Farfantepenaeus paulensis, detergents.

\section{INTRODUÇÃO}

Vários estudos têm demonstrado que, em rios e lagos, detergentes sintéticos são responsáveis por sérios distúrbios ecológicos (APHA, 1992), afetando de maneira significativa os organismos aquáticos, inclusive camarões (Barbieri et al., 2002). Os camarões têm importante papel nos ecossistemas em que vivem e, além disso, são fontes de recursos protéicos para a alimentação humana. São também bons indicadores da quali-dade da água, sendo, por esse motivo, indicados em experimentos para avaliação de impacto ambiental (Harding, 1992).
Poucas são as espécies aquáticas da região neotropical que têm sua sensibilidade determinada. Essa falta de dados de parâmetros toxicológicos exige a utilização de dados bibliográficos sobre a toxicidade de determinados efluentes em condições ambientais muito diferentes das encontradas no Brasil (Araujo \& Nascimento, 1999). Verifica-se, portanto, a necessidade de determinação dos níveis tóxicos de diversas substâncias em espécies nativas, principalmente as de referência, como é o caso do DSS (Dodecil Sulfonato de Sódio). Esses dados são de extremo interesse tanto nos programas de agentes tóxicos como na avaliação de possíveis impactos ambientais

*Corresponding author: Edison Barbieri, e-mail: edisonbarbieri@yahoo.com.br. 
de substâncias tóxicas sobre a biota aquática e suas possíveis implicações na preservação do meio ambiente.

Nos últimos anos, vários estudos têm sido realizados para determinar a toxicidade aguda de diversos compostos químicos para muitas espécies de organismos (Abel, 1974; Terzic \& Abel, 1974; Mittal \& Garg, 1994; Tollefsen, 1998; Damato \& Barbieri, 2003; Barbieri et al., 2005). Entretanto, há poucas alternativas para o biomonitoramento ambiental em regiões tropicais, utilizando-se espécie nativa, em testes de toxicidade com substâncias de referência. No caso do camarão é dada grande atenção aos espécimes cultiváveis, levando-se em consideração parâmetros ambientais como: $\mathrm{pH}$, salinidade, oxigênio dissolvido, concentração de amônia, etc. Neste trabalho determinou-se a toxicidade do LAS-C12 sobre o camarão-rosa (Farfantepenaeus paulensis), uma vez que este detergente é importante poluidor de águas estuarinas, onde esta espécie tem parte do seu ciclo de vida. Determinou-se também a toxicidade aguda do DSS, uma vez que este tensoativo é utilizado amplamente como substância de referência em testes de toxicidade e pela falta de dados sobre toxicidade referentes a espécies nativas brasileiras.

\section{MATERIAL E MÉTODOS}

Os métodos para o desenvolvimento dos testes de toxicidade seguiram os protocolos descritos em APHA (1992). Os testes utilizando larvas de Farfantepenaeus paulensis ( $\mathrm{PL}_{35}$ ) foram realizados em sistema semi-estástico com a renovação da soluçãoteste a cada 24 horas. A água de diluição utilizada foi água do mar filtrada, e para a análise da toxicidade aguda do DSS foram empregadas as seguintes concentrações: 0,0 (controle); 1,0; 2,0; 4,$0 ; 8,0 ; 16$; e $20 \mathrm{mg} \mathrm{L}^{-1}$. Já para a análise da toxicidade aguda do LAS-C12 foram empregadas as seguintes concentrações: 0,0 (controle); 0,$1 ; 0,5 ; 1,0 ; 2,0 ; 4,0 ; 8,0 ;$ e $16 \mathrm{mg} \mathrm{L}^{-1}$. A escolha das concentrações baseou-se em testes prévios realizados com o objetivo de determinar a maior e a menor concentração, distribuindo-se as demais concentrações dentro desses limites. Todos os aquários de 30 litros foram monitorados nos instantes: 0 ; $2 ; 4 ; 6 ; 8 ; 12 ; 24 ; 48 ; 72$; e 96 horas, em relação aos seguintes parâmetros físicos, químicos e biológicos: temperatura, oxigênio dissolvido, $\mathrm{pH}$, salinidade e mortalidade dos animais. Os valores de pH situaram-se entre 8,18 e 8,20 em todos os ensaios. As variações dos níveis de oxigênio dissolvido nos controles durante os ensaios situaram-se entre 7,6 e $7,7 \mathrm{mg} \mathrm{O}_{2} \mathrm{~L}^{-1}$. O controle de temperatura durante os testes foi amplamente atingido, situando-se entre $25,0 \mathrm{e}$ $25,6^{\circ} \mathrm{C}$, e a salinidade situou-se entre 35 e 36 .

A análise biométrica dos espécimes de Farfantepenaeus paulensis revelou que as médias de comprimento situaram-se entre 8 e $10 \mathrm{~mm}$. Utilizaram-se 45 camarões para cada concentração com três réplicas de 15 indivíduos cada, totalizando 630 indivíduos em todo o experimento. Para a avaliação do parâmetro mortalidade dos organismos foram determinadas a CL(I $)_{50} 24 \mathrm{~h}, \mathrm{CL}(\mathrm{I})_{50} 48 \mathrm{~h}$, $\mathrm{CL}(\mathrm{I})_{50} 72 \mathrm{~h} \mathrm{CL}(\mathrm{I})_{50} 96 \mathrm{~h}$ isto é, concentração nominal do agente tóxico que causa mortalidade a $50 \%$ dos organismos em 24, 48, 72 e 96 horas de exposição nas condições de teste. A expressão dos valores nominais com a notação (I) segue a proposta de Lloyd \& Tobby (1979). Foram considerados os testes em que a mortalidade no controle não foi superior a $10 \%$ dos organismos testados.

Empregaram-se 80 indivíduos, com médias de 0,89 ( \pm $0,25)$ grama e $1,1( \pm 0,36)$ centímetro, para avaliar o efeito agudo do DSS e LAS-C12 no metabolismo de rotina do camarão-rosa. Foram feitas medições do consumo de oxigênio utilizando respirômetros selados. Os camarões foram aclimatados à temperatura de $25^{\circ} \mathrm{C}$ e à salinidade de $36 \%$, por 5 dias. Dez camarões para cada concentração de $\operatorname{DSS}\left(0,0 \mathrm{mg} \mathrm{L}^{-1} ; 1,0 \mathrm{mg} \mathrm{L}^{-1} ; 2,5 \mathrm{mg} \mathrm{L}^{-1}\right.$; e $\left.10 \mathrm{mg} \mathrm{L}^{-1}\right)$ e LAS-C12. (0,0 mg L ${ }^{-1} ; 1,0 \mathrm{mg} \mathrm{L}^{-1} ; 2,5 \mathrm{mg} \mathrm{L}^{-1}$; e 5,0 $\mathrm{mg} \mathrm{L}^{-}$

${ }^{1}$ ) foram submetidos à medição do consumo de oxigênio em uma das quatro concentrações.

Antes do início dos experimentos os animais foram mantidos no respirômetro com circulação contínua de água por, pelo menos, 90 minutos, para atenuar o estresse decorrente do manuseio. Em seguida, o fornecimento de água foi suspenso e o respirômetro foi fechado para que o camarão consumisse o oxigênio presente no volume conhecido de água, por um período de uma hora. Os respirômetros foram protegidos por um anteparo para isolar os animais de possíveis movimentações no laboratório. A diferença entre as concentrações de oxigênio, determinadas no início e no final do confinamento, representa o consumo do animal durante o período. Para minimizar o efeito da falta de oxigênio sobre o metabolismo, a duração dos experimentos foi regulada de tal modo que a concentração de oxigênio no final dos experimentos fosse sempre maior do que $70 \%$ de sua concentração inicial. O oxigênio dissolvido foi determinado através do método de Winkler.

Quanto à adição do volume de DSS e LAS-C12, esses foram calculados para cada respirômetro respeitando as diferenças de volume interno de cada um deles. O volume específico de DSS e LAS-C12 referente a um respirômetro foi adicionado com auxílio de uma micropipeta, tomando-se as devidas precauções para evitar o derrame e eventual contaminação da água circulante do sistema. Esses procedimentos foram realizados ao final do período de aclimatação, e em seguida o orifício de entrada de água foi selado com um batoque plástico.

O processamento e tabelamento dos dados foram realizados com auxílio de planilhas eletrônicas, adaptada para o nosso trabalho. Os dados dos experimentos com comparações múltiplas foram submetidos à análise de variância (ANOVA) seguida do teste de Tukey com nível de significância de 0,05 .

\section{RESULTADOS}

Os resultados obtidos para os testes de toxicidade do DSS estão apresentados na Tabela 1. Nos testes toxicológicos com DSS para Farfantepenaeus paulensis constatou-se grande semelhança entre as três réplicas, e os resultados foram 
reprodutíveis para os diversos períodos de exposição. Os valores médios obtidos $\mathrm{CL}(\mathrm{I})_{50}$ para o DSS situaram-se entre: 12,93 e $14,56 \mathrm{mg} \mathrm{L}^{-1}$, para o período de exposição de 24 horas; 11,47 e $12,57 \mathrm{mg} \mathrm{L}^{-1}$, para 48 horas; 9,62 e $10,41 \mathrm{mg} \mathrm{L}^{-1}$, para 72 horas; e 7,94 e 9,33 $\mathrm{mg} \mathrm{L}^{-1}$, para 96 horas, respectivamente.

Nos ensaios com LAS-C12 para $F$. paulensis, também houve grande semelhança entre as três réplicas, e os resultados foram reprodutíveis para os diversos períodos de exposição (Tabela 2). Os valores médios obtidos CL(I) 50 para o LAS-C12 situaram-se entre: 5,93 e $6,82 \mathrm{mg} \mathrm{L}^{-1}$, para o período de exposição de 24 horas; 5,12 e $5,47 \mathrm{mg} \mathrm{L}^{-1}$, para 48 horas; 4,51 e $4,81 \mathrm{mg} \mathrm{L}^{-1}$, para 72 horas; e $4,13 \mathrm{e}$ $4,42 \mathrm{mg} \mathrm{L}^{-1}$, para 96 horas, respectivamente.

As médias da toxicidade aguda do DSS para larvas dos camarões-rosa expostos às diferentes concentrações foram: 13,66 $\mathrm{mg} \mathrm{L}^{-1}$, para o período de exposição de 24 horas; $11,89 \mathrm{mg} \mathrm{L}^{-1}$, para 48 horas; $9,98 \mathrm{mg} \mathrm{L}^{-1}$, para 72 horas; e $8,66 \mathrm{mg} \mathrm{L}^{-1}$, para 96 horas, respectivamente. Já para o LAS-C12 situaram-se entre: 6,37 $\mathrm{mg} \mathrm{L}^{-1}$, para o período de exposição de 24 horas; $5,32 \mathrm{mg} \mathrm{L}^{-1}$, para 48 horas; 4,61 $\mathrm{mg} \mathrm{L}^{-1}$, para 72 horas; e $4,25 \mathrm{mg} \mathrm{L}^{-1}$, para 96 horas, respectivamente (Tabela 3 ).

O consumo específico de oxigênio para os camarões aclimatados à temperatura de $25^{\circ} \mathrm{C}$ variou em função do aumento da concentração de DSS e LAS-C12. O consumo específico de oxigênio diminuiu à medida que a concentração de DSS e LAS-C12 aumentou (Figuras 1 e 2).

As médias do consumo específico de oxigênio para os camarões do grupo controle foram, respectivamente, 0,0084
$\mathrm{mLO}_{2} \mathrm{~g}^{-1} \mathrm{~min}^{-1}$ para o DSS e $0,0096 \mathrm{mLO}_{2} \mathrm{~g}^{-1} \mathrm{~min}^{-1}$ para o LAS-C12. Na maior concentração de DSS $\left(10 \mathrm{mg} \mathrm{L}^{-1}\right)$ empregada a média de consumo foi de $0,0043 \mathrm{mLO}_{2} \mathrm{~g}^{-1} \mathrm{~min}^{-1}$ (Figura 1) e de LAS-C12 (5,0 mg L $\left.{ }^{-1}\right)$ foi de $0,0037 \mathrm{mLO}_{2} \mathrm{~g}^{-1}$ $\min ^{-1}$ (Figura 2). A diminuição das médias dos consumos específicos de oxigênio à concentração de $5,0 \mathrm{mg} \mathrm{L}^{-1}$ de LAS$\mathrm{C} 12$ e $10 \mathrm{mg} \mathrm{L}^{-1}$ para DSS representam, respectivamente, uma baixa da taxa metabólica da ordem de $38,54 \%$ e 51,19\%, respecti-vamente, em relação aos controles.

Utilizando o teste estatístico ANOVA (Tukey, $\mathrm{p}<0,05$ ), constatou-se que as médias do consumo específico de oxigênio à concentração de $10 \mathrm{mg} \mathrm{L}^{-1}$ e $5,0 \mathrm{mg} \mathrm{L}^{-1}$ de DSS e $2,5 \mathrm{mg} \mathrm{L}^{-1}$ e 5,0 $\mathrm{mg} \mathrm{L}^{-1}$ de LAS-C12 são significativamente diferentes em relação aos respectivos controles $\left(0,0 \mathrm{mg} \mathrm{L}^{-1}\right)$. Para a concentração de 1,0 $\mathrm{mg} \mathrm{L}^{-1}$ não houve diferenças significativas.

\section{DISCUSSÃO}

Os efeitos dos poluentes nos organismos vivos são, em geral, muito complexos, sendo resultantes de interferências tanto físico-químicas no meio externo quanto das suas atividades vitais. Por esse motivo, para a monitoração da qualidade ambiental é necessário que se avaliem efeitos sobre os organismos com implicações ecológicas importantes, mas que sejam, ao mesmo tempo, rapidamente identificáveis. Testes de CL50, por exemplo, que quantificam alterações nas taxas de mortalidades sob influências deletérias são amplamente empregados para esse fim (Barbieri et al., 2004a).

Tabela 1 - Valores da toxicidade aguda do DSS sobre o Farfantepenaeus paulensis.

\begin{tabular}{cccc}
\hline & $\begin{array}{c}\text { Valores de CL(I)50 } \\
\text { mg L }^{-1}\end{array}$ & \multicolumn{2}{c}{$\begin{array}{c}\text { Intervalo de confiança } \\
\text { Limite inferior }\end{array}$} \\
\hline Réplica 1 & & \\
$24 \mathrm{~h}$ & 13,50 & 14,10 & 12,90 \\
$48 \mathrm{~h}$ & 11,65 & 12,10 & 11,20 \\
$72 \mathrm{~h}$ & 9,91 & 10,51 & 9,31 \\
$96 \mathrm{~h}$ & 8,72 & 9,27 & 8,17 \\
\hline Réplica 2 & & & 14,06 \\
$24 \mathrm{~h}$ & 14,56 & 14,91 & 11,87 \\
$48 \mathrm{~h}$ & 12,57 & 13,27 & 9,71 \\
$72 \mathrm{~h}$ & 10,41 & 11,11 & 8,83 \\
$96 \mathrm{~h}$ & 9,33 & 9,83 & 12,48 \\
Réplica 3 & & & 10,98 \\
$24 \mathrm{~h}$ & 12,93 & 13,38 & 9,32 \\
$48 \mathrm{~h}$ & 11,47 & 11,96 & 7,27 \\
$72 \mathrm{~h}$ & 9,62 & 9,92 & \\
$96 \mathrm{~h}$ & 7,94 & 8,61 & \\
\hline
\end{tabular}


Tabela 2 - Valores da toxicidade aguda do LAS-C12 sobre o Farfantepenaeus paulensis.

\begin{tabular}{cccc}
\hline & \multicolumn{2}{c}{ Valores de CL(I)50 } & \multicolumn{2}{c}{ Intervalo de confiança } \\
& mg L $^{-\mathbf{1}}$ & Limite inferior & Limite superior \\
\hline Réplica 1 & & & \\
$24 \mathrm{~h}$ & 6,82 & 7,22 & 7,42 \\
$48 \mathrm{~h}$ & 5,12 & 5,42 & 4,82 \\
$72 \mathrm{~h}$ & 4,81 & 5,11 & 4,51 \\
$96 \mathrm{~h}$ & 4,42 & 4,62 & 4,22 \\
\hline Réplica 2 & & & \\
$24 \mathrm{~h}$ & 6,36 & 6,66 & 6,06 \\
$48 \mathrm{~h}$ & 5,47 & 5,77 & 5,17 \\
$72 \mathrm{~h}$ & 4,51 & 4,71 & 4,31 \\
$96 \mathrm{~h}$ & 4,13 & 4,17 & 4,09 \\
\hline Réplica 3 & & & \\
$24 \mathrm{~h}$ & 5,93 & 6,63 & 5,23 \\
$48 \mathrm{~h}$ & 5,27 & 5,47 & 5,07 \\
$72 \mathrm{~h}$ & 4,52 & 4,72 & 4,32 \\
$96 \mathrm{~h}$ & 4,20 & 4,25 & 4,15 \\
\hline
\end{tabular}

Tabela 3 - Médias dos respectivos $\mathrm{CL}_{50}$ para o SDS e LAS-C12 sobre Farfantepenaeus paulens.

Os testes de toxicidade constituem o mais comum, mais prático, mais rápido e mais barato instrumento de determinação de toxicidade de um efluente (Harding, 1992), podendo, com as mesmas características, ser aplicados às variações de riscos e ao diagnóstico de qualidade dos corpos receptores (Barbieri et al., 2005). Um importante fato no uso adequado desses testes para o diagnóstico e previsão de riscos é sua sensibilidade, que é baseada não apenas na sensibilidade da espécie, mas também nas condições de realização do teste (Araujo \& Nascimento, 1999; Barbieri, 2007). Utilizando-se ensaios padronizados pode-se então comparálos para selecionar o melhor instrumento de controle ambiental.

A sensibilidade individual de um organismo é influenciada decisivamente pela idade do indivíduo, pois com o envelhecimento ocorrem modificações na atividade de diversas enzimas (Swedmark et al., 1971; Barbieri et al., 2002). Por este motivo, larvas de camarão são geralmente mais sensíveis a certas toxinas do que os adultos. Nos primeiros estágios de desenvolvimento os organismos apresentam maior sensibilidade, devido, prova- velmente, à maior atividade mitótica durante essas fases (Araujo \& Nascimento, 1999). Além disso, na idade avançada a sensibilidade pode diferir bastante da média de toxicidade de certo composto químico. A capacidade de absorção reduzida do estômago e do intestino pode diminuir a sensibilidade a algumas substâncias tóxicas (Tollefsen, 1998; Barbieri et al., 2003). É mais comum, contudo, verificar que camarões com mais idade se mostram mais sensíveis a poluentes químicos do ambiente, pois nestes crustáceos a metabolização e excreção dos contaminantes se processam de modo mais lento, permanecendo durante mais tempo a toxina no organismo.

O ensaio realizado com o camarão-rosa neste trabalho é considerado um teste agudo envolvendo um caráter letal. Expressamos os resultados deste trabalho como $\mathrm{CL}_{50} \mathrm{em}$ vez de utilizarmos CENO ou NOEC (concentração do efeito não observado) porque a proposta foi o desenvolvimento de metodologia e o estabelecimento e a sensibilidade destes métodos a tóxicos de referência. 


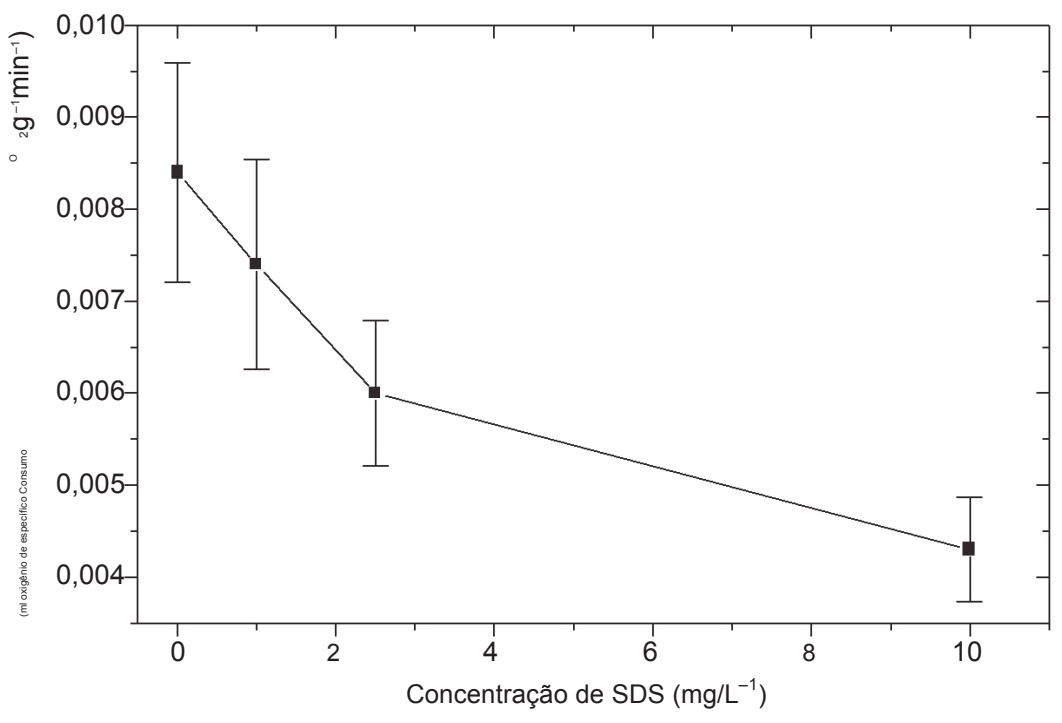

Figura 1 - Consumo específico de oxigênio para Farfantepenaeus paulens em relação ao aumento da concentração de DSS. As barras são os respectivos desvios-padrão de cinco amostras.

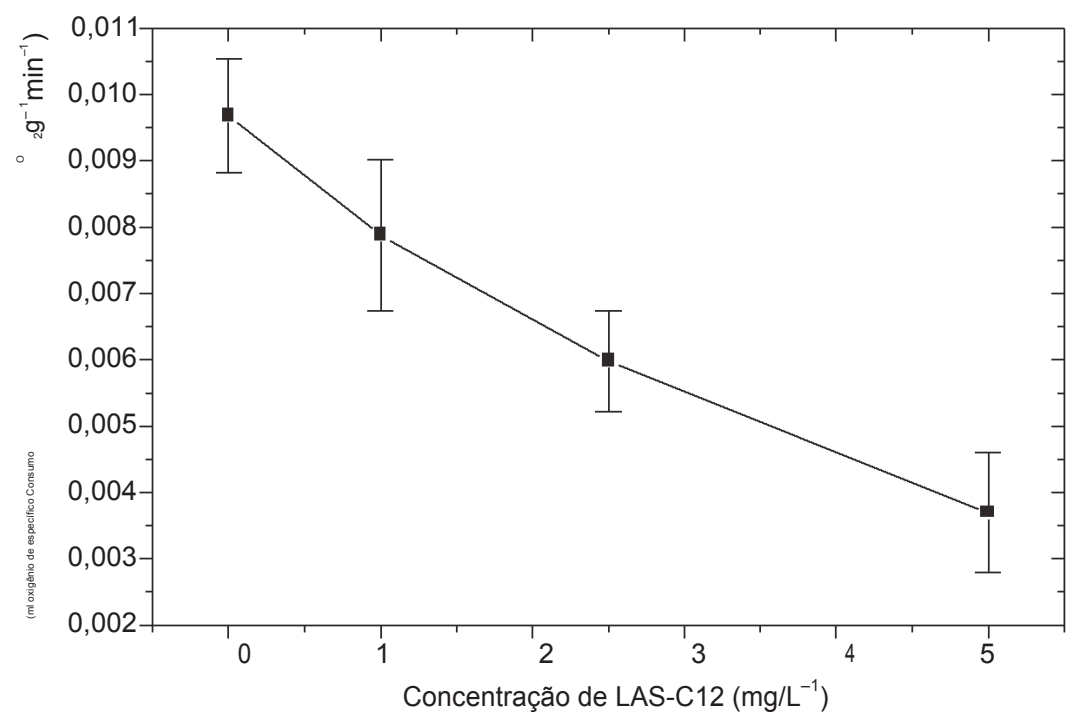

Figura 2 - Consumo específico de oxigênio para Farfantepenaeus paulens em relação ao aumento da concentração de LAS-C12. As barras são os respectivos desvios-padrão de cinco amostras.

Considerando-se o país como um todo, a utilização de detergentes sintéticos ainda não atingiu, no Brasil, as mesmas proporções que os caracterizam como agentes poluidores responsáveis pelo controle da qualidade do meio ambiente no que diz respeito à poluição por detergentes em determinadas localidades e à necessidade de exercer ação preventiva visando evitar problemas futuros. Estudo realizado pelo Laboratório de Controle da Qualidade de Saneantes e Cosméticos da Univer- sidade Estadual do Rio de Janeiro, em 1993, apurou que, dentre os 15 produtos de diferentes marcas analisados, 9 não eram biodegradáveis (Vieira, 1993). Esses produtos, em vez de utilizarem o LAS como princípio ativo, estavam utilizando o ABS, que é biorresistente. Na indústria, o LAS é freqüentemente empregado na fabricação de detergentes sintéticos e sabões em pó, pois, apesar de ser mais tóxico do que o ABS (Abel, 1974), permanece por menos tempo na natureza. 
Os surfactantes agem sobre a fertilidade, desenvolvimento embrionário, reprodução, hábitos alimentares e comportamento de animais aquáticos (Mittal \& Garg, 1994; Barbieri et al., 2004b). Mastroti (1997) evidenciou comprometimento significativo no desenvolvimento dos embriões de ouriços-do-mar (Lytechinus varigatus) submetidos a $0,13 \mathrm{mg} \mathrm{L}^{-1}$ de LAS-C12. Trabalhos realizados pela Sociedade Japonesa de Testes Químicos (JCTS, 1987) relatam que os valores de $\mathrm{CL}_{50}$ para o camarão Penaeus japonicus exposto ao LAS foram de $0,96 \mathrm{mg} \mathrm{L}^{-1}$ para o estágio de mísis; $1,43 \mathrm{mg} \mathrm{L}^{-1}$ para pós-larvas de um dia $\left(\mathrm{PL}_{1}\right) ; 1,80 \mathrm{mg} \mathrm{L}^{-1}$ para $\mathrm{PL}_{14} ;$ e $6,24 \mathrm{mg} \mathrm{L}^{-1}$ para $\mathrm{PL}_{45}$. Estes resultados são semelhantes aos encontrados neste trabalho para F. paulensis. Supriyono et al. (1998), estudando indivíduos no estágio $\mathrm{PL}_{33}$ dessa mesma espécie expostos ao LAS-C12, encontraram $\mathrm{CL}_{50}$ de 4,24 $\mathrm{mg} \mathrm{L}^{-1}$, após 96 horas de exposição. Estes resultados sugerem que, à medida que as larvas do camarão crescem, tornam-se mais resistentes ao LAS.

Wasielesky (2000) observou que, após 30 dias de exposição à amônia, a sobrevivência de $F$. paulensis no nível de segurança utilizado por esse autor não apresentou diferença significativas em relação ao controle. No entanto, os resultados, em termos de crescimento em peso úmido e biomassa final, mostraram que até mesmo uma concentração tão baixa de 0,91 $\mathrm{mg} \mathrm{L}^{-1}$ de amônia, que equivaleu a $1 / 4$ do nível de segurança determinado por Ostrensky \& Wasielesky (1995) para Farfantepenaeus paulensis, causou retardo nos níveis de crescimento da mesma. Por este motivo sugerimos futuros trabalhos com concentrações menores que as letais e com períodos de exposição do LAS maiores.

\section{CONCLUSÃO}

Os resultados da toxicidade do DSS para o camarão-rosa revelaram que as $\mathrm{CL}(\mathrm{I})_{50}$ em seus períodos de exposição estudados (24, 48, 72 e 96 horas) apresentavam sobreposição dos intervalos de confiança entre si, indicando que os resultados eram reprodutíveis. Para o LAS-C12, as CL(I) 50 de 4,42 e 4,13 $\mathrm{mg} \mathrm{L}^{-1}$ registradas para o camarão-rosa neste trabalho, são muito preocupantes, uma vez que concentrações de tensoativos aniônicos superiores a estas foram encontradas em trabalhos realizados na costa brasileira. Assim, fica evidente a necessidade de realização de outros trabalhos utilizando outras concentrações de LAS, bem como outras espécies, para o biomonitoramento de águas estuarinas. Em relação às taxas metabólicas, o consumo específico de oxigênio diminuiu à medida que a concentração de DSS e LAS-C12 aumentou.

\section{REFERÊNCIAS BIBLIOGRÁFICAS}

ABEL, P. D., 1974, Toxicity of synthetic detergent to fish and aquatic invertebrates. J. Fish Biol., 6: 279-298.

APHA, 1992, American Public Health Association. Standard methods for examination of water and wastewater. 18 ed. Washington: APHA, 1992.

ARAUJO, M. M. S. \& NASCIMENTO, I. A., 1999, Testes ecotoxicológicos marinhos: análise de sensibilidade. Ecotoxicol. Environ. Restora., 2(1): 41-47.
BARBIERI, E., OLIVEIRA, I. R. \& SERRALHEIRO, P. A C., 2002, The use of metabolism to evaluate the toxicity of dodecil benzen sodium sulfonate (LAS-C12) on the Mugil platanus according to the temperature and salinity. J. Exp. Mar. Biol. Ecol., 277: 109-127.

BARBIERI, E., DAMATO, M., PHAN, V. N. \& GOMES, V., 2003, Toxicidade aguda do LAS-C12 sobre juvenis de tainha (Mugil platanus). Mundo da Saúde, 27(4): 571-578.

BARBIERI, E., GARCIA, C. A. B. \& ALVES, J. P. H., 2004a, Utilização do camarão sete-barbas (Xiphopenaeus kroyeri) na determinação da toxicidade da água de produção de petróleo caracterizada no Estado de Sergipe. Mundo da Saúde, 28(4): 421-430.

BARBIERI, E., GARCIA, C. A. B., AlvES, J. P. H., ARAG̃̃O, K. A. \& DÓREA, H., 2004b, Saúde ambiental: emprego de Poecilia vivípara e Artemia salina para determinar a toxicidade aguda para a água de produção de petróleo caracterizada no Estado de Sergipe. Mundo da Saúde, 29(2): 213-225.

BARBIERI, E., PASSOS, A. E. \& GARCIA, C. A. B., 2005, Use of metabolism to evaluate the subletal toxicity of mercury on Farfantepanaeus brasiliensis larvae. Journal of Shellfish Research, 24(4): 608-611.

BARBIERI, E., 2007, Use of oxygen consumption and ammonium excretion to evaluate the sublethal toxicity of cadmium and zinc on litopenaeus schmitti (Burkenroad, 1936, Crustacea). Water Environ. Res., 79(6): 641-646.

DAMATO, M. \& BARBIERI, E., 2003, Emprego de uma espécie indicadora sul-americana na determinação da toxicidade aguda para o cobre, zinco, níquel e alumínio. Mundo da Saúde, 27(4): 551-558.

HARDING, L. E., 1992, Measures of marine environmental quality. Mar. Pollut. Bull., 25(1-4): 23-27.

LLOYD, R. \& TOOBY, T. E., 1979, New terminology required for short-term static fish bioassay LC(I)50. Bull Environm. Contam. Toxicol., 22(1-3).

MASTROTI, R. R., 1997, Toxicidade e biodegradabilidade de tensoativos aniônicos em água do mar. Dissertação de mestrado, Dpto. Oceano. Biol., Inst. Oceanogra., Universidade de São Paulo.

MITTAL, A. K. \& GARG, T. K., 1994, Effect of an anionic detergentsodium dodecyl sulphate exposure on club cells in the epidermis of Clarias batrachus. J. Fish Biol., 44: 857-875.

OSTRENSKY, A. \& WASIELESKY, W. Jr., 1995, Acute oxicity of amonia to varius life atrages of the São Paulo shrimp Panaeus paulensis. Aquaculture, 132: 339-347

SUPRIYONO, E., TAKASHIMA, F. \& STRUSSMANN, C. A., 1998, Toxicity of Linear alkylbenzene sulphonate (LAS) to juvenile kuruma shrimp, Penaeus japonicus: a histopathological study on acute and subchronic levels. J. Tokyo Univ. Fish., 85(1):1-10.

SWEDMARK, M., BRAATEN, B., EMANUELSSON, E. \& GRANMO, A., 1971, Biological effects of surface active agents on marine animals. Mar. Biol., 9: 183-201.

TERZIC, S. \& ABEL, M., 1974, Imput and behaviour of linear alkybenzenesulphonates (LAS) in a stratifield estuary. Mar. Pollut. Bull., 28(12): 735-740.

TOLLEFSEN, K. E., INGEBRIGTSEN, K., OLSEN, A. J., ZACHARIASSEN, K. E. \& JOHNSEN, E. S., 1998, Acute and toxicokinetics of 4-heptyphenol in juvenile Atlantic cod (Gadus morhua L.). Environ. Toxicol. Chem., 17(4): 740-746.

VIEIRA, C. L., 1993, Mar de espumas. Rev. Ciência Hoje, 16(95): 3-9.

WASIELESKY, W. JR., 2000, Cultivo de juvenis de camarão-rosa no estuário da Lagoa dos Patos. Tese de doutorado, Fundação Universidade do Rio Grande, 98p. 